\title{
Design principles for biochemical oscillations with limited energy resources
}

\author{
Zhiyu Cao $\odot$, Huijun Jiang $\odot{ }^{*}$ and Zhonghuai Hou $\odot^{\dagger}$ \\ Department of Chemical Physics and Hefei National Laboratory for Physical Sciences at Microscales, \\ iChEM, University of Science and Technology of China, Hefei, Anhui 230026, China
}

(Received 2 April 2020; accepted 12 November 2020; published 7 December 2020)

\begin{abstract}
As biochemical systems may frequently suffer from limited energy resources, so that internal molecular fluctuation has to be utilized to induce random rhythm, it is still a great theoretical challenge to understand the elementary principles for biochemical systems with limited energy resources to maintain phase accuracy and phase sensitivity. Here, we address the issue by deriving the energy-accuracy and the sensitivity-accuracy trade-off relations for a general biochemical model, analytically and numerically. We find that biochemical systems have a much lower energy cost by using noise-induced oscillations to keep almost equal efficiency to maintain precise processes compared with the energy cost using normal oscillations, clearly elucidating a survival mechanism when energy resources are limited. Moreover, an optimal system size is predicted where both the highest sensitivity and highest accuracy can be reached at the same time, providing a new strategy for the design of biological networks with limited energy sources.
\end{abstract}

DOI: 10.1103/PhysRevResearch.2.043331

For a living system to survive and grow, it needs to meet certain regulatory functions and sensory adaptations with energy constantly injected and dissipated. In particular, for biochemical oscillations which are crucial in controlling the timing of life processes, such as the cell cycle, circadian clocks, and glycolysis, both accurate determination of the period and sensitive response to external signals are expected to be ensured [1-5]. Recently, several findings have implied that there may be some underlying trade-off relations preventing them from been reached simultaneously [6-10]. For instance, Lan et al. proposed a general relation between energy dissipation rate, adaptation speed, and maximum adaptation accuracy to study cost-performance trade-offs [6]. Understanding such relations is then of great importance to uncover design principles for biochemical oscillations to maintain enhanced phase accuracy of the internal period and phase sensitivity to external signals. So far, it has been revealed that for a biochemical oscillation system with sufficient energy supplies, additional energy exceeding a critical value can be used to enhance the system's phase accuracy and phase sensitivity [11-14]. However, biochemical oscillation systems in the real world may frequently suffer from limited energy resources [15-17], so that the critical energy to maintain the oscillatory behavior may even be lacking and internal molecular fluctuation has to be utilized to induce random rhythm. It is then still a great theoretical challenge to understand the elementary principles

\footnotetext{
*hjjiang3@ustc.edu.cn

†hzhlj@ustc.edu.cn
}

Published by the American Physical Society under the terms of the Creative Commons Attribution 4.0 International license. Further distribution of this work must maintain attribution to the author $(s)$ and the published article's title, journal citation, and DOI. for biochemical systems with limited energy resources to maintain phase accuracy and phase sensitivity.

Here, we address the issue by studying the trade-off relations for a general biochemical model theoretically, starting from which both normal oscillations for sufficient energy sources and noise-induced oscillations for limited energy supplies can be described well in a unified theoretical framework. By applying the concepts of stochastic thermodynamics as well as the phase reduction method, the energy-accuracy and the sensitivity-accuracy trade-off relations are finally derived, which provide general design principles for biochemical oscillations. Application of these principles shows that biochemical systems can keep almost equal efficiency to maintain precise processes at much lower energy cost by using noise-induced oscillations for limited energy resources compared with the efficiency achieved by using normal oscillations for sufficient energy supplies. Moreover, an optimal system size is found where both high sensitivity and high accuracy can be reached at the same time, predicting a new design strategy for biological networks with limited energy sources.

For a general biochemical system of size $V$ including $N$ well-stirred species and $M$ reactions $\left(R_{1}, \ldots, R_{M}\right)$, its dynamics can be described by the chemical Langevin equation (CLE), which is expected to be satisfied for biochemical reaction systems with mesoscopic system size which ensures the existence of a "macro-infinitesimal time scale" [18] as

$\dot{x}_{j}=\sum_{\rho=1}^{M} v_{\rho}^{j} w_{\rho}(\boldsymbol{x})+\frac{1}{\sqrt{V}} \sum_{\rho=1}^{M} v_{\rho}^{j} \sqrt{w_{\rho}(\boldsymbol{x})} \xi_{\rho}(t), \quad j=1, \ldots, N$.

where $\boldsymbol{x}=\left(x_{1}, \ldots, x_{N}\right)^{\mathrm{T}}$ is the concentration vector, $v_{\rho}^{j}$ is the stoichiometric coefficient of $x_{j}$ in reaction $R_{\rho}$, 
$w_{\rho}(\boldsymbol{x})$ is the transition probability, and $\boldsymbol{\xi}(t)$ is independent Gaussian white noises with zero mean and time correlation $\left\langle\xi_{\rho}(t) \xi_{\rho^{\prime}}(s)\right\rangle=\delta_{\rho \rho^{\prime}} \delta(t-s)$. The corresponding chemical Fokker-Planck equation (CFPE) [19] is then $\partial_{t} p(\boldsymbol{x}, \tau)=$ $-\sum_{i} \partial_{x_{i}}\left[f_{i}(\boldsymbol{x}) p(\boldsymbol{x}, \tau)\right]+(1 / 2) \sum_{i, j} V \partial_{x_{i}} \partial_{x_{j}}\left[G_{i j}(\boldsymbol{x}) p(\boldsymbol{x}, \tau)\right]$, where $p(\boldsymbol{x}, \tau)$ is the time-varying probability density function, $G_{i j}(\boldsymbol{x})=\sum_{\rho=1}^{M} v_{\rho}^{i} v_{\rho}^{j} w_{\rho}(\boldsymbol{x})$, and the drift $f_{j}(\boldsymbol{x})=$ $\sum_{\rho=1}^{M} v_{\rho}^{j} w_{\rho}(x)$ is the macroscopic rate under the thermodynamic limit $V \gg 1$. If there is a Hopf bifurcation for the systems as some control parameters change, biochemical oscillation will occur. Above the bifurcation, a normal oscillation will be observed; however, near but below the bifurcation, stochastic oscillation can emerge due to the internal noise. A general theoretical description of the oscillation dynamics including both normal oscillation and noise-induced oscillation (NIO) [20,21] can be achieved by the stochastic normal form theory we established before [18,22,23]; that is, the time evolution of oscillation amplitude $r$ and phase $\theta$ is

$$
\begin{aligned}
& \dot{r}=\alpha r+C_{r} r^{3}+\frac{\varepsilon^{2}}{2 V r}+\frac{\varepsilon}{\sqrt{V}} \eta_{r}(t), \\
& \dot{\theta}=\omega+C_{i} r^{2}+\frac{\varepsilon}{r \sqrt{V}} \eta_{\theta}(t),
\end{aligned}
$$

where $C_{r}<0$ and $C_{i}$ are constants, $\varepsilon^{2}$ is the averaged noise intensity, $\eta_{r}(t)$ and $\eta_{\theta}(t)$ are the averaged independent Gaussian white noises with unit variances (see details in Appendix A). Specially, $\alpha$ can be related to the energy resources [16] which determine the oscillatory behaviors of the systems. For large enough $V$, normal oscillations with amplitude $r_{m}^{2}=-\frac{\alpha}{C_{r}}+$ $\frac{\varepsilon^{2}}{2 \alpha} V^{-1}+o\left(V^{-2}\right)$ can be observed when $\alpha>0$. When $\alpha<0$, the energy resources are not enough to support normal oscillations. However, there is still a nonzero-amplitude solution $r_{m}^{2}=-\frac{\varepsilon^{2}}{2 \alpha} V^{-1}+o\left(V^{-2}\right)$, indicating that the internal noise could be utilized to induce stochastic rhythms to maintain the system's function $[17,24]$ in such a situation. Biochemical systems need to maintain highly accurate oscillations, which is crucial in controlling the timing of life processes [11]. The energy-accuracy trade-off relation describes the constraint as to how accurate a biochemical oscillator can be with given energy sources. As the time translation symmetry of the bi. lend\{equation \}ochemical system is inherently broken, the phase of the oscillation exhibits a diffusive behavior. The coefficient of phase diffusion $D_{\theta}$ can be used to measure the accuracy of the oscillators. From Eqs. (2) and (3), one can derive that the steady-state probability distribution reads $p_{s}(r)=C_{0} r \exp \left[\left(\alpha r^{2}+\frac{1}{2} C_{r} r^{4}\right) /\left(\varepsilon^{2} / V\right)\right]$, with $C_{0}$ being a normalization constant. Notice that there is an attractor of limit cycle and the system will fluctuate aroundaround it due to internal noise. Then, one can calculate the mean and variance of $\theta$, i.e., $\langle\theta(t)\rangle \simeq\left(\omega+C_{i} r_{m}^{2}\right) t \equiv$ $\omega_{s} t$ and $\left\langle\theta(t)^{2}\right\rangle-\langle\theta(t)\rangle^{2} \simeq \varepsilon^{2} t /\left(V r_{m}^{2}\right)$, where $\omega_{s}=\omega+C_{i} r_{m}^{2}$ is the effective phase angular velocity of the attractor. In fact, here we use the approximations that $\left\langle r^{2}\right\rangle \simeq r_{m}^{2}$ and $\left\langle r^{-1}\right\rangle \simeq r_{m}^{-1}$ which hold near the Hopf bifurcation. Thus the diffusion constant of the phase fluctuation is given by

$$
D_{\theta}=\varepsilon^{2} /\left(V r_{m}^{2}\right)
$$

Besides, the energy dissipation critical for living systems to realize special functions (such as correcting errors in biomolecular recognition or improving robustness in cell development [6]) can be directly related to the violation of detailed balance in nonequilibrium systems and determined by computing the entropy production in the underlying reaction network. The explicit expression for energy dissipation in one cycle of the oscillation is $\Delta W=\dot{S}_{\text {tot }} T_{\text {cyc }}$, with $\dot{S}_{\text {tot }}$ being the total entropy production rate and $T_{\text {cyc }}=2 \pi / \omega_{s}$ being the period of the oscillation. At last, by applying the concepts of stochastic thermodynamics [25], the total entropy production rate is found to be $\dot{S}_{\text {tot }}=\left(k_{b}+k_{a} V r_{m}^{2}\right) /(2 \pi)$, where $k_{a}, k_{b}$ are system-size-dependent parameters (see Appendix B for details). Now, we arrive at the first main result of this paper, namely, the energy-accuracy trade-off relation. (Under the condition of the selected parameters, the period hardly changes with the parameter value, especially in the noise-induced oscillation region. So we did not consider the accuracy dimensionless to the period; see Appendix B for details.)

$$
D_{\theta}= \begin{cases}\frac{k_{a} \varepsilon^{2}}{\omega}\left(\Delta W+\frac{k_{b} C_{r}}{C_{i} \alpha-C_{r} \omega}\right)^{-1}+D_{\theta,-}^{s}, & \alpha<0 \\ \frac{k_{a} \varepsilon^{2}}{\omega}\left(\Delta W-\frac{k_{b}}{\omega V}\right)^{-1}+D_{\theta,+}^{s}, & \alpha>0 .\end{cases}
$$

Here, Eq. (5) provides a general design principle to quantitatively describe the balance between energy cost and phase accuracy for not only normal oscillations $(\alpha>0)$ but also NIOs $(\alpha<0)$.

Several conclusions can be obtained. Firstly, Eq. (5) can recover the reported phase diffusion for normal oscillations [11] $D_{\theta}=W_{0} /\left(\Delta W-W_{c}\right)$ with $W_{0}=k_{a} \varepsilon^{2} / \omega, W_{c}=$ $-k_{b} C_{r} /\left(C_{i} \alpha-C_{r} \omega\right)$ and an additional $D_{\theta}^{s}\left[\right.$ for $\alpha>0, D_{\theta,-}^{s}=$ $-C_{i} \varepsilon^{2} /(\omega V)$; for $\left.\alpha<0, D_{\theta,+}^{s}=-2 \alpha\right]$. Secondly, Eq. (5) shows that phase diffusion can be suppressed by increasing thermodynamic cost $\Delta W$, while there is always a minimal phase diffusion constant $D_{\theta}^{s}$ that cannot be completely eliminated even for infinite cost.

Thirdly, as $D_{\theta}=\varepsilon^{2} /\left(V r_{m}^{2}\right)$, the scaling law for the energy cost $\Delta W$ of the system size $V$ holds near the Hopf bifurcation point as

$$
\Delta W=n+m V^{v}, \begin{cases}v=0, n=\frac{2 k_{b} \alpha-k_{a} \varepsilon^{2}}{2 \alpha \omega}, m=0, & \alpha<0 \\ v=\frac{1}{2}, n=0, m=\frac{k_{a} \varepsilon}{\omega} \sqrt{-\frac{2}{C_{r}}}, & \alpha=0 \\ v=1, n=0, m=-\frac{\alpha k_{a}}{C_{r} \omega}, & \alpha>0\end{cases}
$$

that is, for normal oscillations, $\Delta W$ increases linearly as $V$ increases, while for NIOs $\Delta W$ is independent of the system size $V$. Remarkably, biochemical systems may have to optimize their ability to maintain accurate oscillations under a given energy cost [26]. For instance, the molecular motor needs to deliver the cargo at a high speed and in a punctual manner with low energy dissipation [27]. Thus a transport efficiency $\eta_{T}$ [26] quantitatively describing such ability can be derived from the energy-accuracy trade-off relation [Eq. (5)] via the thermodynamic uncertainty relation (TUR; see Appendix B for details) [28-30]

$$
\eta_{T}=\frac{\langle\dot{\theta}\rangle^{2}}{D_{\theta} \dot{S}_{\text {tot }}}
$$


More importantly, according to Eqs. (6) and (7), it will be found that biochemical systems have significantly lower energy cost to maintain accuracy for NIOs than for normal oscillations, which will be elucidated more clearly in the numerical simulations.

As biochemical oscillations with high sensitivity are vulnerable to external perturbation and fluctuation, accuracy and sensitivity can be treated as two trade-off properties $[13,14,31,32]$. Here, the sensitivity $\chi$ is characterized by the ability of the biochemical circuits to respond to external signals. It can be obtained by comparing the phase shift after delivering a perturbation [33-35] and calculated from the phase response curve (PRC) function [13] as follows. The deterministic evolution equation of Eq. (1) can be expressed as $\dot{\phi}=\nabla_{\boldsymbol{x}} \phi \cdot \boldsymbol{f}(\boldsymbol{x})$. In the presence of an external signal $\boldsymbol{\beta}(t)$, the deterministic term obeys $\boldsymbol{f}_{k}(\boldsymbol{x})=\boldsymbol{f}(\boldsymbol{x})+k \boldsymbol{\beta}(t)$, where $k$ is the parameter to be perturbed. Then, the PRC function reads $Z_{k}(\phi)=\nabla_{x} \phi \cdot \boldsymbol{\beta}(t)$, and the sensitivity $\chi$ can be obtained as the normalized value of the signal-independent factor $\nabla_{x} \phi[36,37]$ at $r=r_{m}$ (see Appendix C for details),

$$
\chi_{m}=\chi\left(r_{m}\right)=\frac{\left|C_{i}\right| r_{m}^{2}}{\sqrt{\left(\alpha^{2}-2 C_{r} \varepsilon^{2} / V\right)}} .
$$

Based on Eq. (8), we can derive the second main result of this paper,

$$
2 \log \chi_{m}^{*}=C_{0}+\log D_{\theta},
$$

with $C_{0}=\log \left\{2 \pi C_{i}^{2} r_{m}^{4} /\left[\varepsilon^{2} k_{a}\left(\alpha^{2}-2 C_{r} \varepsilon^{2} / V\right)\right]\right\}$ and $\chi_{m}^{*}=$ $\chi_{m} / \sqrt{\dot{S}_{\text {tot }}}$. Equation (9) shows that an increase in phase accuracy $D_{\theta}^{-1}$ cannot be accompanied by an increase in the normalized phase sensitivity $\chi_{m}^{*}$, which can be treated as the sensitivity-accuracy trade-off relation under fixed energy conditions [see Appendix C for detailed analysis of Eq. (9)]. Such a trade-off relationship always holds when system parameters change, providing another design principle for biochemical systems to measure the balance between the sensory adaptation to external signals and the regulation of the internal oscillation. More interestingly, we can also obtain a scaling law for phase sensitivity $\chi_{m}$ of the system size $V$ as

$$
\chi_{m} \sim V^{\kappa}, \begin{cases}\kappa=-1, & \alpha<0 \\ \kappa=0, & \alpha \geqslant 0 .\end{cases}
$$

For a biochemical oscillation system, it is efficient to maintain high regulation of the internal oscillation and, simultaneously, sensitive adaptation to external signals. Then, a dynamic efficiency $\eta_{S}$ can be properly defined as the ratio of the sensitivity and phase fluctuation, i.e., $\chi_{m}^{2} / D_{\theta}$, to quantitatively describe such ability as

$$
\eta_{S}=\frac{C_{i}^{2} V r_{m}^{6}}{\varepsilon^{2}\left(\alpha^{2}-2 C_{r} \varepsilon^{2} / V\right)} .
$$

The obtained dynamic efficiency can further be related to the information inequality $v_{k, \phi}^{2} / D_{\theta} \leqslant \dot{\mathbb{D}}_{P E}$, where $v_{k, \phi}$ is the change rate of the current difference $\langle\phi\rangle_{k}-\langle\phi\rangle$ (here, $\langle\phi\rangle$ is the mean of phase for the original dynamics, and $\langle\phi\rangle_{k}$ is the mean for the dynamics perturbed by the signal) and $\mathbb{D}_{P E}$ is the Pearson divergence between the original dynamics and the perturbed dynamics, which show similar evolutionary behavior to the total entropy production [14]. The quantity $v_{k, \phi}$ is proportional to the system sensitivity, and $\dot{\mathbb{D}}_{P E}$ determines the upper bound of the dynamic efficiency. Moreover, as the total entropy production rate hardly changes with system size in NIOs, we find that the dynamic efficiency can approach its upper bound by adjusting the size parameter $V$ of biochemical oscillation systems. Therefore one can design a biochemical system to enhance the sensitivity and reduce the fluctuation simultaneously by changing its internal properties to maximize $\eta_{S}$. Interestingly, an optimal system size $V_{\text {opt }} \approx$ $-\frac{3 C_{r} \varepsilon^{2}}{4 \alpha^{2}}$ can be achieved by setting $\partial\left(\eta_{S}\right) / \partial V=0$, where biochemical systems with limited energy sources can reach their best performance of both high sensitivity and high accuracy. Furthermore, the scaling law between $V_{\text {opt }}$ and $\alpha$ reads as $V_{\text {opt }} \sim \alpha^{-2}$.

Now, we apply the above analytical results to a well-known biochemical oscillation system, the Brusselator model, involving two distinct biochemical species $X_{1}, X_{2}$ and four reaction channels

$$
\begin{gathered}
A \rightarrow X_{1}, \quad B+X_{1} \rightarrow X_{2}, \\
X_{1} \rightarrow C, \quad 2 X_{1}+X_{2} \rightarrow 3 X_{1} .
\end{gathered}
$$

Here, $\boldsymbol{w}=\left(A, B X_{1}, X_{1}, X_{1}^{2} X_{2}\right)$ represent the corresponding transition rates. In the thermodynamic limit where the internal noise terms can be ignored, a supercritical Hopf bifurcation occurs for $\alpha=\left(B-B_{c}\right) / 2$ with $B_{c}=A^{2}+1$. We numerically simulate Eq. (1) by Euler methods with a time step of $10^{-4}$. After a long enough transition time, $10^{5}$ trajectories are used
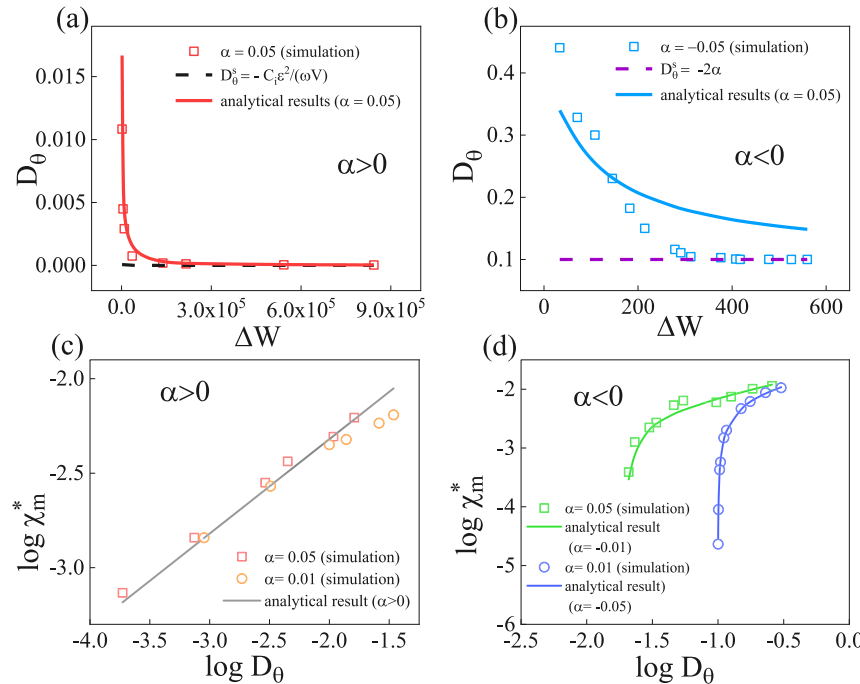

FIG. 1. Trade-off relations for biochemical oscillations with sufficient $(\alpha>0)$ or limited $(\alpha<0)$ energy sources. (a) and (b) The simulated energy-accuracy trade-off relation for $\alpha>0$ and $\alpha<0$ shows that the phase fluctuation constant $D_{\theta}$ decreases as the freeenergy cost $\Delta W$ increases, which agrees well with analytical curves fitted according to Eq. (5). Besides, there is always a minimal phase diffusion constant $D_{\theta}^{s}$ [the black dashed line for $\alpha>0$ in (a) and the purple dashed line for $\alpha<0$ in (b)] that cannot be completely eliminated even for infinite cost. (c) and (d) The simulated sensitivity-accuracy trade-off relation for $\alpha>0$ and $\alpha<0$ shows that phase sensitivity $\chi_{m}$ also increases as the phase diffusion constant $D_{\theta}$ increases. 

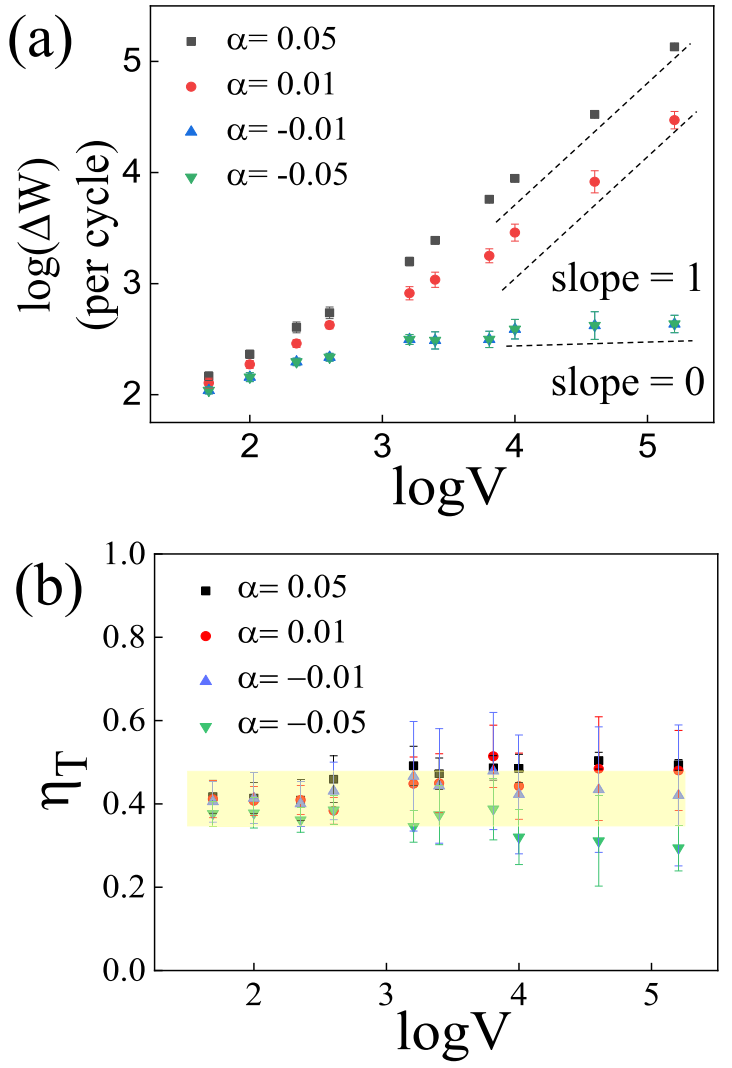

FIG. 2. Biochemical systems with limited energy sources $(\alpha<0)$ need significantly less energy to maintain accuracy than systems with sufficient sources $(\alpha>0)$. (a) Scaling law between energy cost per cycle $\Delta W$ and system size $V$. (b) Transport efficiency $\eta_{T}$ for different system sizes $V$. Error bars in (a) and (b) represent standard deviations. For fixed $V, \eta_{T}$ is robust against changes in $\alpha$ and $V$, while $\Delta W$ is much smaller for $\alpha<0$ than for $\alpha>0$.

to calculate the energy cost $\Delta W$. Other details of the model and parameters can be found in Appendix D.

Both of the trade-off relations [Eqs. (5) and (9)] are shown in Fig. 1. It can be observed that the phase diffusion constant $D_{\theta}$ is inversely proportional to the energy cost $\Delta W$, confirming that the accuracy-energy trade-off relation holds for both NIOs and normal oscillations [Figs. 1(a) and 1(b)]. Similarly, the normalized sensitivity $\chi_{m}^{*}$ also increases as $D_{\theta}$ increases, which verifies the sensitivity-accuracy trade-off relation for both normal oscillations and NIOs [Figs. 1(c) and 1(d)].

Dependence of the energy cost $\Delta W$ and the transport efficiency $\eta_{T}$ on the system size $V$ is shown in Figs. 2(a) and 2(b), respectively. Simulated $\Delta W$ increases proportionally as $V$ increases for normal oscillations and is nearly unchanged for NIOs, which is in good consistence with the scaling law (6) [Fig. 2(a)]. The scaling law when $\alpha=0$ and $\Delta W \sim V^{1 / 2}$ has also been verified. Details are presented in Appendix B. Additionally, as $\eta_{T}$ is almost equal for different types of oscillations [Fig. 2(b)] and $\Delta W$ for NIOs is always smaller than that for normal oscillations for fixed $V$, it thus leads to the quite interesting conclusion that, for NIOs, the system can keep almost the same efficiency to maintain precise processes at much lower energy cost, elucidating clearly the advantage of noise-induced oscillations in limited energy supplies.
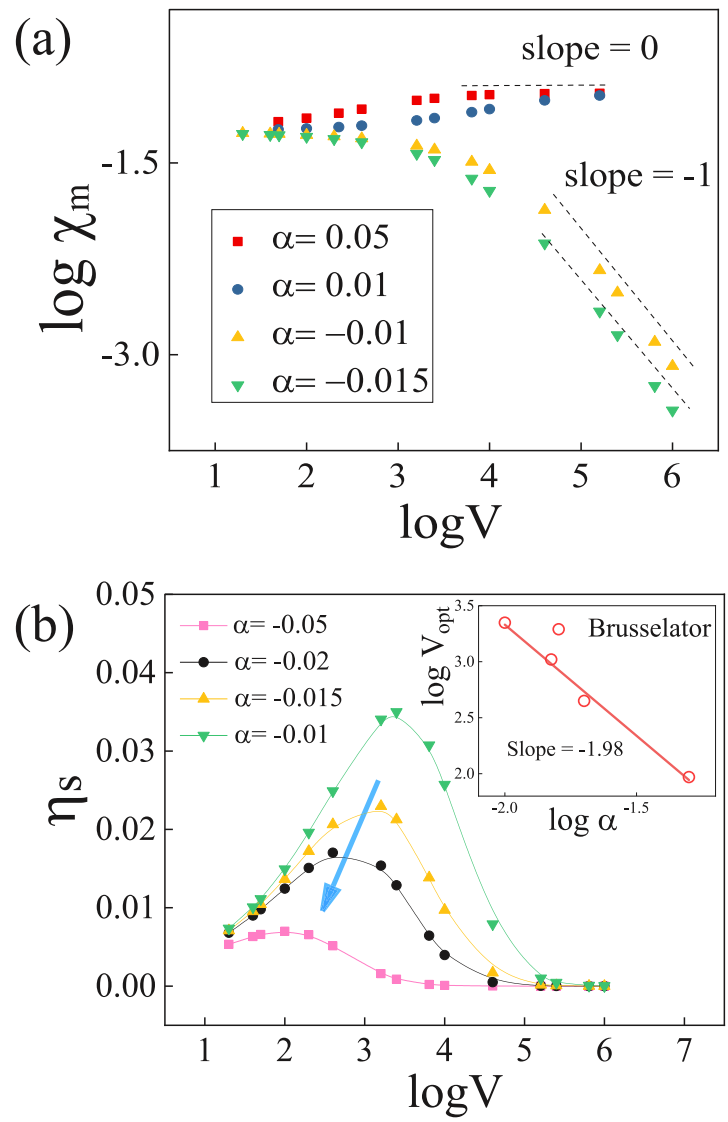

FIG. 3. A new strategy for the design of biological networks in limited energy resources to achieve both high accuracy and high sensitivity. (a) Scaling law between the sensitivity $\chi_{m}$ and the system size $V$ for sufficient $(\alpha>0)$ or limited $(\alpha<0)$ energy sources. (b) Dependence of the dynamic efficiency $\eta_{S}$ on the system size $V$. The dynamic efficiency $\eta_{S}$ for limited energy resources shows a maximum for an optimal system size $V_{\text {opt }}$ where $\chi_{m}$ still changes little. Inset: Explicit dependence and scaling law between $V_{\text {opt }}$ and $\alpha$.

As shown in Fig. 3(a), the phase sensitivity $\chi_{m}$ changes little with increasing system size $V$ for normal oscillations and is inversely proportional to $V$ for NIOs, agreeing with the scaling law [Eq. (10)] very well. Remarkably, the dynamic efficiency $\eta_{S}$ for NIOs in Fig. 3(b) shows a maximum for an optimal system size $V_{\text {opt }}$ where $\chi_{m}$ still changes little. Surely, the system size cannot be adjusted freely. However, biochemical systems are continuously evolving, possibly by adjusting other parameters to make the oscillation systems work at the optimal system size. We need to emphasize that there are many reported findings in the literature indicating that some naturally occurring systems do employ a strategy similar to the one we predict. For instance, it has been reported that ionchannel clusters of an optimal size were the most favorable for intracellular calcium signaling [38-41], and we have also found that the well-known signal-to-noise ratio of the NIO has been found to be maximum in many naturally occurring systems studied in our previous work, such as mammalian circadian oscillation [20] and surface catalytic reactions [17], when the system size is varied. Based on such evidence, we propose that this finding predicts a new strategy for the design 
(a)

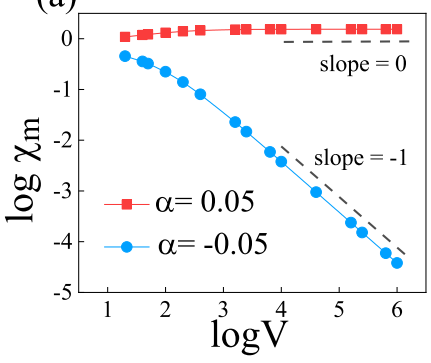

(b)

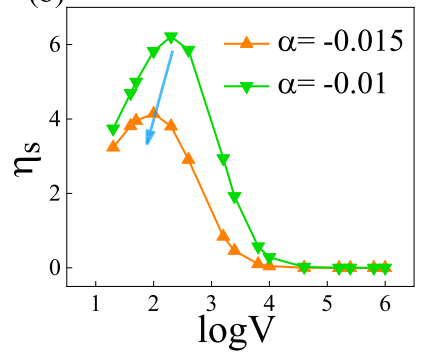

FIG. 4. Further validation of the derived design principle of biological networks in the circadian clock model. (a) Scaling law between the phase sensitivity $\chi_{m}$ and system size $V$. (b) The dynamic efficiency $\eta_{S}$ shows a maximum for an optimal system size $V_{\text {opt }}$.

of biological networks in limited energy resources to achieve both high accuracy and high sensitivity simultaneously, which is absent for systems with sufficient energy supplies.

To further demonstrate the broad application of our design principles, we have also applied them to another important biochemical oscillation, i.e., the circadian clock model, describing how living organisms keep an internal sense of time and adapt their behavior [1]. As shown in Fig. 4, we find that our main results, such as the scaling law [Eq. (10)] and the optimal system size [obtained from Eq. (11)], still hold in the circadian clock model. The phase sensitivity $\chi_{m}$ is nearly independent of the system size $V$ for normal oscillations and is inversely proportional to $V$ for NIOs, also agreeing with the scaling law [Eq. (10)] very well. Furthermore, a maximum of the dynamic efficiency $\eta_{S}$ for NIOs with an optimal system size $V_{\text {opt }}$ has also been found in Fig. 4(b), where $\chi_{m}$ is kept unchanged. Other details of the model and parameters can be found in Appendix D. Notice that other parameters besides the system size have also been included in our approach by $C_{i}, C_{r}$, $\varepsilon$, etc.; thus the proposed design principles are still applicable when other parameters matter.

In conclusion, energy-accuracy and sensitivity-accuracy trade-off relations have been revealed for a general biochemical system by applying the framework of stochastic thermodynamics as well as the phase reduction method. According to these relations, it was found that biochemical systems may maintain their necessary regulatory function via noise-induced oscillation to reduce the energy cost when energy resources are limited. More interestingly, an optimal system size for systems to achieve both high accuracy and high sensitivity has also been derived from the trade-off relations, predicting a new strategy for the design of biological networks with limited energy sources. For oscillations associated with other types of bifurcations, such as relaxation oscillations, the stochastic normal form could be different. Nevertheless, a similar idea and strategy to those of the present work may also be applied [42], which deserves further study. As our findings are of important relevance to many rhythmic processes in biochemical systems and can be extended to other realistic systems straightforwardly, it is our hope that the reported principles will enhance our ability to design new biochemical systems for practical applications.

\section{ACKNOWLEDGMENTS}

This work is supported by MOST (Grants No. 2016YFA0400904 and No. 2018YFA0208702), NSFC (Grants No. 21973085, No. 21833007, No. 21790350, No. 21673212, No. 21521001, and No. 21473165), the Fundamental Research Funds for the Central Universities (Grant No. WK2340000074), and Anhui Initiative in Quantum Information Technologies (Grant No. AHY090200).

\section{APPENDIX A: STOCHASTIC NORMAL FORM THEORY}

We follow the standard procedure to get the normal form. Setting $Z=r e^{i \theta}$,

$$
\begin{aligned}
\frac{d Z}{d t}= & (\alpha+i \omega) Z+\left(C_{r}+i C_{i}\right)|Z|^{2} Z \\
& +\frac{1}{\sqrt{V}} \sum_{\rho}\left(v_{1 \rho}^{\prime}+i v_{2 j \rho}^{\prime}\right) \sqrt{w_{\rho}} \xi_{\rho},
\end{aligned}
$$

where $\boldsymbol{v}_{j \rho}^{\prime}=\left(T^{-1} \boldsymbol{v}\right)_{j \rho} ;$ equivalently,

$$
\frac{d r}{d t}=\left(\alpha r+C_{r} r^{3}\right)+\frac{1}{\sqrt{V}} \sum_{\rho} \chi_{r \rho} \circ \xi_{\rho}
$$

and

$$
\frac{d \theta}{d t}=\left(\omega+C_{i} r^{2}\right)+\frac{1}{\sqrt{V}} \sum_{\rho} \chi_{\theta \rho} \circ \xi_{\rho},
$$

where

$$
\begin{aligned}
& \chi_{r \rho}=\left(v_{1 \rho}^{\prime} \cos \theta+v_{2 j \rho}^{\prime} \sin \theta\right) \sqrt{w_{\rho}}, \\
& \chi_{\theta \rho}=\frac{1}{r}\left(-v_{1 \rho}^{\prime} \sin \theta+v_{2 j \rho}^{\prime} \cos \theta\right) \sqrt{w_{\rho}} .
\end{aligned}
$$

Using the stochastic averaging procedure [43], as a long-time Markov approximation, one can get the following equations:

$$
\frac{d r}{d t}=\alpha r+C_{r} r^{3}+\frac{K(r)}{V}+\frac{\varepsilon_{r}}{\sqrt{V}} \xi_{r}
$$

and

$$
\frac{d \theta}{d t}=\omega+C_{i} r^{2}+\frac{K(\theta)}{V}+\frac{\varepsilon_{\theta}}{r \sqrt{V}} \xi_{\theta},
$$

where

$$
\begin{aligned}
K(r) & =\frac{1}{2 \pi} \sum_{\rho} \int_{0}^{2 \pi} d \theta\left(\chi_{r \rho} \partial_{r} \chi_{r \rho}+\chi_{\theta \rho} \partial_{\theta} \chi_{r \rho}\right), \quad K(\theta) \\
& =\frac{1}{2 \pi} \sum_{\rho} \int_{0}^{2 \pi} d \theta\left(\chi_{r \rho} \partial_{r} \chi_{\theta \rho}+\chi_{\theta \rho} \partial_{\theta} \chi_{\theta \rho}\right) .
\end{aligned}
$$

Here,

$$
\varepsilon_{r}^{2}=\frac{1}{2 \pi} \sum_{\rho} \int_{0}^{2 \pi} d \theta \chi_{r \rho}^{2}, \quad \varepsilon_{\theta}^{2}=\frac{1}{2 \pi} \sum_{\rho} \int_{0}^{2 \pi} d \theta \chi_{\theta \rho}^{2}
$$

are the averaged noise intensities. Moreover, one can expand the reaction rates as

$$
w_{\rho}=\sum_{i+j=0}^{n} w_{\rho}^{i j}(r \cos \theta)^{i}(r \sin \theta)^{j} .
$$


As $r$ is small for the situation when the control parameter is near the Hopf bifurcation point, one can get that $K(\theta)$ is zero [22] and

$$
\varepsilon_{r}^{2}=\varepsilon_{\theta}^{2}=\frac{1}{2} \sum_{\rho}\left[\left(v_{1 \rho}^{\prime}\right)^{2}+\left(v_{2 \rho}^{\prime}\right)^{2}\right] w_{\rho}^{00} .
$$

Neglecting the $i+j \geqslant 2$ terms, one can derive the stochastic normal form equations

$$
\begin{gathered}
\dot{r}=\alpha r+C_{r} r^{3}+\frac{\varepsilon^{2}}{2 V r}+\frac{\varepsilon}{\sqrt{V}} \eta_{r}(t), \\
\dot{\theta}=\omega+C_{i} r^{2}+\frac{\varepsilon}{r \sqrt{V}} \eta_{\theta}(t),
\end{gathered}
$$

where for $\alpha>0$, the amplitude $r_{m}^{2}=$ $-\left(\sqrt{\alpha^{2}-2 C_{r} \varepsilon^{2} / V}+\alpha\right) /\left(2 C_{r}\right) \quad\left(\right.$ when $\left.V \rightarrow \infty, \quad r_{m}^{2} \rightarrow-\frac{\alpha}{C_{r}}\right)$ can be observed. For $\alpha<0$, the energy resources are not enough to support normal oscillation. However, there is still a nonzero-amplitude solution $r_{m}^{2}=$ $-\left(\sqrt{\alpha^{2}-2 C_{r} \varepsilon^{2} / V}+\alpha\right) /\left(2 C_{r}\right)\left(\right.$ when $\left.V \rightarrow \infty, r_{m}^{2} \rightarrow-\frac{\varepsilon^{2}}{2 \alpha V}\right)$.

\section{APPENDIX B: ENERGY-ACCURACY TRADE-OFF RELATION}

\section{Change rate of generalized flux}

The calculation of entropy production is based on the concepts of stochastic thermodynamics [25]. Firstly, one can define the entropy production of the system: $s(\tau)=$ $-\ln p(x, \tau)$, along a stochastic trajectory $\chi(t)=\left\{\left.\boldsymbol{x}(\tau)\right|_{0} ^{t}\right\}$ during a time interval $[0, t]$. Here, the path probability density $p(\boldsymbol{x}, \tau)$, which measures the probability of traversing a given path with initial conditions, is the solution of the Fokker-Planck equation evaluated along the trajectory at time $\tau$. The change rate of the trajectorydependent total entropy production $\dot{s}_{\text {tot }}(\tau)$ can be decomposed into two contributions: $\dot{s}_{\text {tot }}(\tau)=\dot{s}(\tau)+\dot{s}_{m}(\tau)$. We note that the probability current $J_{i}(x, \tau)=\frac{1}{2} \sum_{j} G_{i j}\left(H_{j}-\right.$ $\left.\frac{1}{V} \partial_{x_{j}}\right) p(\boldsymbol{x}, \tau)$, with $H_{j}=2 \sum_{k} \Gamma_{j k} f_{k}^{\prime}(\boldsymbol{G} \boldsymbol{\Gamma}=\boldsymbol{I})$ and $f_{k}^{\prime}=$ $f_{k}-1 /(2 V) \sum_{j}\left(\partial G_{k j}\right) /\left(\partial x_{j}\right)$. The latter term of the total entropy production $\dot{s}_{m}(\tau)=V \sum_{i} H_{i} \dot{x}_{i}$ is related to the change rate of the heat dissipation in the environment with $\dot{q}(\tau)=T \dot{s}_{m}(\tau)$. Averaging $\dot{s}_{\text {tot }}(\tau)$ over the path ensemble, one can obtain that $\dot{S}_{\text {tot }}(\tau)=\left\langle\dot{s}_{\text {tot }}(\tau)\right\rangle=$ $2 V \int d \boldsymbol{x}\left[\left(\sum_{i, j} \Gamma_{i j} J_{i} J_{j}\right) / p(\boldsymbol{x}, \tau)\right]$. Observing the numerator of the integral term, it can be treated as a quadratic form. Moreover, the matrix $\Gamma$ is positive definite, which makes the above quadratic form greater than 0 . Actually, one can turn it into a canonical form as $\sum_{i, j} \Gamma_{i j} J_{i} J_{j}=\boldsymbol{J}^{\mathrm{T}}\left(\boldsymbol{C} \cdot \boldsymbol{C}^{\mathrm{T}}\right)^{-1} \boldsymbol{J} \geqslant 0$. Here, $\boldsymbol{C}(\boldsymbol{x})$ satisfies $\boldsymbol{C}(\boldsymbol{x}) \boldsymbol{C}(\boldsymbol{x})^{\mathrm{T}}=\boldsymbol{G}(\boldsymbol{x})$ [44]. We take a commonly used choice following the seminal paper by Gillespie [45], namely, $C_{j \rho}(\boldsymbol{x})=v_{\rho}^{j} \sqrt{w_{\rho}(\boldsymbol{x})}$. In the steady state, the entropy production rate of the system vanishes. Thus the total entropy production rate equals the entropy flux rate. Based on the stochastic normal form theory, one can get the change rate of any generalized flux [26]: $\Theta[\chi]=V \int \boldsymbol{\Lambda}(\boldsymbol{x})^{\mathrm{T}} \circ \dot{\boldsymbol{x}} d t$, where $\boldsymbol{\Lambda}(\boldsymbol{x})$ is a projection operator with a projection matrix $\boldsymbol{Q}$ such that $\boldsymbol{\Lambda}=\boldsymbol{Q} \cdot \boldsymbol{f}$. We assume that the system has equilibrium points $\boldsymbol{x}_{s}$ satisfying $f_{i}\left(\boldsymbol{x}_{s}\right)=0$ for $\forall i=1, \ldots, N$ and a Hopf bifurcation value $\mu_{c}$ (denotes the control parame- ter) [46]. The Jacobian matrix $\boldsymbol{\Phi}$, with $\Phi_{i j}=\left.\left(\partial_{x_{j}} f_{i}\right)\right|_{x=x_{s}}$, has two conjugate eigenvalues $\lambda_{ \pm}=\alpha(\mu) \pm i \omega$, with $\alpha\left(\mu_{c}\right)=0$. The other $N-2$ eigenvalues of $\boldsymbol{\Phi}$ all have strictly negative real parts with absolute values considerably larger than 0 . By variable transformation $\boldsymbol{u}=\boldsymbol{T}^{-1}\left(\boldsymbol{x}-\boldsymbol{x}_{s}\right)$, the linear part can be transformed to Jordan form, which reads $\dot{\boldsymbol{u}}=\Lambda_{H B} \boldsymbol{u}+$ $\mathcal{O}\left(\boldsymbol{u}^{2}\right)+\frac{1}{\sqrt{V}} \eta(t)$. Here, $\boldsymbol{\eta}(t)=\boldsymbol{T}^{-1} \boldsymbol{\zeta}\left(\boldsymbol{x}_{s}, t\right)$ is the noisy term with $\zeta_{j}(t)=\sum_{\rho} v_{\rho}^{j} \sqrt{w_{\rho}(\boldsymbol{x})} \xi_{\rho}(t)$. The variances of $\boldsymbol{\eta}(t)$ satisfy $\left\langle\eta_{i}(t) \eta_{j}(s)\right\rangle=2 D_{i j} \delta(t-s)$, where $\boldsymbol{D}=\boldsymbol{T}^{-1} \boldsymbol{G}\left(\boldsymbol{T}^{-1}\right)^{\mathrm{T}}$. The change rate of currents near the Hopf bifurcation can be calculated by both spatial and temporal average:

$$
\begin{aligned}
\langle\dot{\Theta}\rangle & =\lim _{t \rightarrow \infty} \frac{\langle\Theta\rangle}{t}=V\left\langle\boldsymbol{\Lambda}^{\mathrm{T}} \dot{\boldsymbol{x}}\right\rangle_{s} \\
& =V\left\langle\boldsymbol{f}^{\mathrm{T}} \boldsymbol{Q}^{\mathrm{T}} \dot{\boldsymbol{x}}\right\rangle_{s}=2 V \sum_{i, j} R_{i j}\left\langle u_{i} \dot{u}_{j}\right\rangle_{s},
\end{aligned}
$$

where $\boldsymbol{R}^{T}=\boldsymbol{T}^{\mathrm{T}} \boldsymbol{\Phi}^{\mathrm{T}} \boldsymbol{Q}^{\mathrm{T}} \boldsymbol{T}, h_{i j}=\left\langle u_{i} \dot{u}_{j}\right\rangle_{s}$, and we have used $\boldsymbol{f}(\boldsymbol{x})=\boldsymbol{f}\left(\boldsymbol{x}_{s}\right)+\boldsymbol{\Phi}\left(\boldsymbol{x}-\boldsymbol{x}_{s}\right)+o\left(|\boldsymbol{u}|^{2}\right) \simeq \boldsymbol{\Phi} \boldsymbol{T} \boldsymbol{u}$. Note that in the stationary state, $h_{i j}=-h_{j i}$ and $h_{i i}=0$.

$$
\begin{gathered}
h_{12} \simeq \frac{1}{2} \omega\left\langle r^{2}\right\rangle_{s}, \\
h_{1 j} \simeq 0, \quad h_{2 j} \simeq 0 \quad(j \geqslant 3) .
\end{gathered}
$$

For $i, j \geqslant 3$,

$$
h_{k j}=\frac{\left(\lambda_{k}-\lambda_{j}\right) D_{k j}}{\left(\lambda_{k}+\lambda_{j}\right) V} .
$$

Thus

$$
\begin{aligned}
\langle\dot{\Theta}\rangle & =2 V \sum_{i, j} R_{i j}\left\langle u_{i} \dot{u}_{j}\right\rangle_{s} \\
& =V\left(R_{12}-R_{21}\right) \omega\left\langle r^{2}\right\rangle_{s}+2 \sum_{j, k \geqslant 3} R_{k j} D_{k j} \frac{\lambda_{k}-\lambda_{j}}{\lambda_{k}+\lambda_{j}} .
\end{aligned}
$$

We approximate $\left\langle r^{2}\right\rangle_{s} \simeq r_{m}^{2}$, and when $r=r_{m}$, the probability distribution function becomes maximum; thus

$$
\langle\dot{\Theta}\rangle \simeq \frac{\left(R_{12}-R_{21}\right) \omega}{2} V r_{m}^{2}+\text { const. }
$$

In particular, Eq. (B6) can be used to calculate the change rate of entropy flux by choosing $\Lambda_{i}=H_{i}$.

\section{Entropy production}

The change rate of entropy production of the system is given by

$$
\begin{aligned}
\dot{s}(\tau)= & -\partial_{\tau} p(\boldsymbol{x}, \tau)-\frac{1}{p(\boldsymbol{x}, \tau)} \sum_{i} \partial_{x_{i}} p(\boldsymbol{x}, \tau) \dot{x}_{i} \\
= & {\left[-\partial_{\tau} p(\boldsymbol{x}, \tau)+\left.\frac{2 V}{p(\boldsymbol{x}, \tau)} \sum_{i, j} \Gamma_{i j} J_{j}\right|_{\boldsymbol{x}(\tau)} \dot{x}_{i}\right] } \\
& -V \sum_{i} H_{i} \dot{x}_{i} .
\end{aligned}
$$


The ensemble-averaged total entropy production rate can be calculated as

$$
\begin{aligned}
\dot{S}_{\mathrm{tot}}(\tau) & =\left\langle\dot{s}_{\mathrm{tot}}(\tau)\right\rangle \\
& =\left\langle\left.\frac{2 V}{p(\boldsymbol{x}, \tau)} \sum_{i, j} \Gamma_{i j} J_{j}\right|_{\boldsymbol{x}(\tau)} \dot{x}_{i}\right\rangle \\
& =2 V \int d \boldsymbol{x} \frac{\left[\sum_{i, j} \Gamma_{i j} J_{i} J_{j}\right]}{p(\boldsymbol{x}, \tau)} .
\end{aligned}
$$

We set $\boldsymbol{F}=\boldsymbol{C}^{-1}$, and $\boldsymbol{J}^{F}=\boldsymbol{F} \boldsymbol{J}$ with $i$ th component $J_{i}^{F}=$ $\sum_{j} J_{i} F_{j i}$. Then,

$$
\begin{aligned}
\sum_{i, j} \Gamma_{i j} J_{i} J_{j} & =\boldsymbol{J}^{\mathrm{T}} \boldsymbol{\Gamma} \boldsymbol{J} \\
& =\boldsymbol{J}^{\mathrm{T}} \boldsymbol{G}^{-1} \boldsymbol{J} \\
& =\boldsymbol{J}^{\mathrm{T}}\left(\boldsymbol{C} \cdot \boldsymbol{C}^{\mathrm{T}}\right)^{-1} \boldsymbol{J} \\
& =\left(\boldsymbol{C}^{-1} \boldsymbol{J}\right)^{\mathrm{T}} \cdot\left(\boldsymbol{C}^{-1} \boldsymbol{J}\right) \\
& =(\boldsymbol{F} \boldsymbol{J})^{\mathrm{T}} \cdot(\boldsymbol{F} \boldsymbol{J}) \\
& =\sum_{i}\left(\sum_{j} J_{i} F_{j i}\right)^{2}=\sum_{i}\left(J_{i}^{F}\right)^{2} \geqslant 0 .
\end{aligned}
$$

Thus the second law is obtained,

$$
\dot{S}_{\text {tot }}(\tau) \geqslant 0,
$$

which is a defining feature of the system.

\section{Scaling law and TUR}

When $V$ is large enough, different scaling laws can be readily seen in the following equations:

$$
D_{\theta}= \begin{cases}-2 \alpha, & \alpha<0 \\ \sqrt{-\frac{C_{r} \varepsilon^{2}}{2 V}}, & \alpha=0 \\ -\frac{\varepsilon^{2} C_{r}}{V \alpha}, & \alpha>0\end{cases}
$$

and

$$
\Delta W= \begin{cases}\frac{2 k_{b} \alpha-k_{a} \varepsilon^{2}}{2 \alpha \omega}, & \alpha<0 \\ \frac{k_{a} \varepsilon}{\omega} \sqrt{-\frac{2 V}{C_{r}}}, & \alpha=0 \\ -\frac{\alpha k_{a} V}{C_{r} \omega}, & \alpha>0 .\end{cases}
$$

The thermodynamic uncertainty relation for $\theta$ reads (when $t \rightarrow \infty)$

$$
\frac{\operatorname{Var}[\theta] \cdot S_{\mathrm{tot}}}{\langle\theta\rangle^{2}} \simeq \frac{\varepsilon^{2}\left(L_{12}-L_{21}\right) \omega}{\omega_{s}^{2}} .
$$

Here, we need to emphasize that Eq. (B13) holds for both $\alpha>0$ and $\alpha<0$. In addition, we plot the scaling law for the energy cost per cycle $\Delta W \sim V^{1 / 2}$ when $\alpha=0$ in Fig. 5. The simulation results show great agreement with our theoretical predictions.

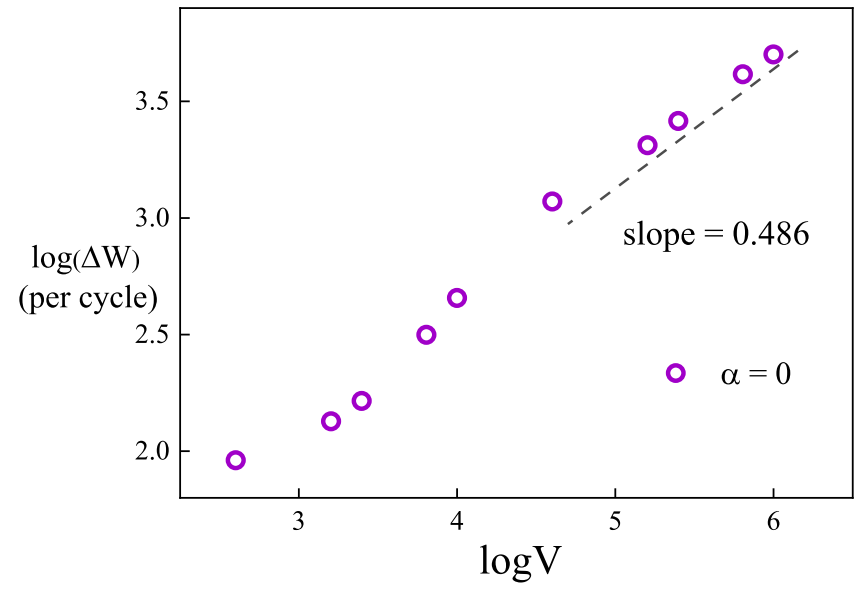

FIG. 5. Scaling law for the energy dissipation per cycle $\Delta W$ when $\alpha=0$. The scaling law between energy cost per cycle and system size has been plotted. The simulation results verify our theoretical predictions, $\Delta W \sim V^{1 / 2}$.

\section{APPENDIX C: SENSITIVITY-ACCURACY TRADE-OFF RELATION}

\section{Phase response curve}

The deterministic evolution equation of the chemical Langevin equation can be expressed as

$$
\dot{\phi}=\Omega=\nabla_{x} \phi \cdot f(x) .
$$

In the presence of an external signal, the dynamics obey the following equation:

$$
f_{k}(\boldsymbol{x})=f(\boldsymbol{x})+k \boldsymbol{\beta}(t),
$$

where $\boldsymbol{\beta}(t)=\left\{\beta_{1}(t), \ldots, \beta_{N}(t)\right\}^{\mathrm{T}}$ is used to describe external signals and $k$ is the parameter to be perturbed. After perturbation, the phase evolution equation becomes [37]

$$
\begin{aligned}
\dot{\phi} & =\nabla_{\boldsymbol{x}} \phi \cdot[f(\boldsymbol{x})+k \boldsymbol{\beta}(t)] \\
& =\Omega+k Z_{k}(\phi),
\end{aligned}
$$

where

$$
Z_{k}(\phi)=\nabla_{x} \phi \cdot \boldsymbol{\beta}(t),
$$

known as the phase response curve (PRC) function. The PRC function is obtained by comparing the phase shift after delivering a perturbation at a given duration of time.

\section{Isochron of the limit cycle}

To get the sensitivity-accuracy trade-off relation, we first use the stochastic normal form theory to analyze the isochron structure of the limit cycle [13]. Considering the radial evolution of the orbit outside the deterministic limit cycle with initial starting point $\left(r_{0}, \theta_{0}\right)$, we have [here, we set $\alpha_{c}=$ $\sqrt{\alpha^{2}-2 \varepsilon^{2} C_{r} / V}$ and $\left.y_{1}=\left(\sqrt{\alpha^{2}-2 C_{r} \varepsilon^{2} / V}-\alpha\right) / 2 C_{r}\right]$

$$
r(t)^{2}=y_{1}-\frac{\alpha_{c} / C_{r}}{1+C_{1} e^{-2 \mu t}},
$$

where $C_{1}$ is a constant determined by the initial condition

$$
-\frac{\alpha_{c} / C_{r}}{r_{0}^{2}-y_{1}}=1+C_{1} \text {. }
$$


(a)

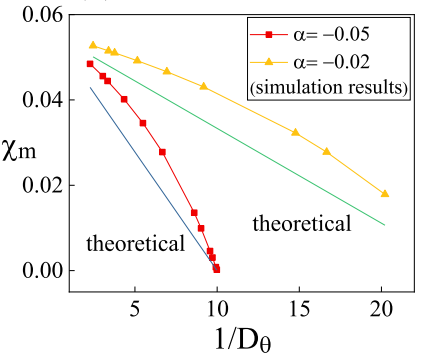

(b)

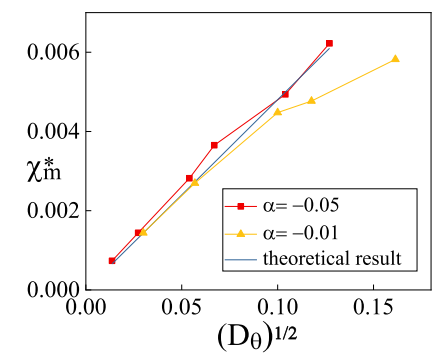

FIG. 6. (a) and (b) Trade-off relation between phase sensitivity and accuracy for NIOs. Both theoretical and simulation results show that in all oscillation regions, phase sensitivity $\chi_{m}$ decreases as the accuracy $D_{\theta}$ increases.

Suppose the point eventually approaches the limit cycle and converges with the point of the initial phase value. This leads to the result

$$
\begin{aligned}
\theta & =\theta_{0}+C_{i} \int_{0}^{\infty}\left(r^{2}-r_{s}^{2}\right) d t \\
& =\theta_{0}-\frac{C_{i}}{2 C_{r}} \ln \left(r_{0}^{2}-y_{1}\right)+\frac{C_{i}}{2 C_{r}} \ln \left(-\frac{\mu}{C_{r}}\right) .
\end{aligned}
$$

Thus, the isochron can be obtained as

$$
\phi(r, \theta)=\theta-\frac{C_{i}}{2 C_{r}} \ln \left(r^{2}-y_{1}\right)+\frac{C_{i}}{2 C_{r}} \ln \left(-\frac{\mu}{C_{r}}\right) .
$$

\section{Scaling law and trade-off relation}

When $V$ is large enough, different scaling laws can be readily seen in the following equations:

$$
\chi_{m}= \begin{cases}-\frac{C_{i} \varepsilon^{2}}{2 \alpha^{2} V}, & \alpha<0 \\ \frac{C_{i}}{C_{r}}, & \alpha \geqslant 0 .\end{cases}
$$

Moreover, to get the trade-off relation, we can make the approximation by Taylor expansion, which reads, for $\alpha<0$,

$$
r_{m}^{2} \simeq-\frac{1}{2 \alpha}\left(\frac{\varepsilon^{2}}{V}+\frac{C_{r} \varepsilon^{4}}{2 \alpha^{2} V^{2}}\right) .
$$

Then,

$$
\frac{1}{D_{\theta}}=-\frac{1}{2 \alpha}-\frac{C_{r} \varepsilon^{2}}{2 \alpha^{3} V}
$$

Thus

$$
\chi_{m}=\frac{C_{i}}{2 C_{r}}+\frac{\alpha C_{i}}{C_{r}} \frac{1}{D_{\theta}} .
$$

For $\alpha<0, \dot{S}_{\text {tot }}$ shows little difference for different system sizes $V$ due to the scaling law, and the sensitivity-accuracy trade-off relation reads $\chi_{m} \simeq C_{i} /\left(2 C_{r}\right)+\alpha C_{i} /\left(C_{r} D_{\theta}\right)$. Since $\alpha C_{i} / C_{r}<0$, phase sensitivity decreases monotonically as phase accuracy decreases. For $\alpha>0$, the sensitivityaccuracy trade-off relation reads $\chi_{m}^{*} \simeq \sqrt{Q_{s} D_{\theta}}$, where $Q_{s}=$ $k_{a} \varepsilon^{2} C_{r}^{2} /\left(2 \pi C_{i}^{2}\right)$ is a constant independent of system size $V$ and resource supply $\alpha$. Thus phase sensitivity decreases monotonically as phase accuracy decreases under fixed energy conditions (Fig. 6).

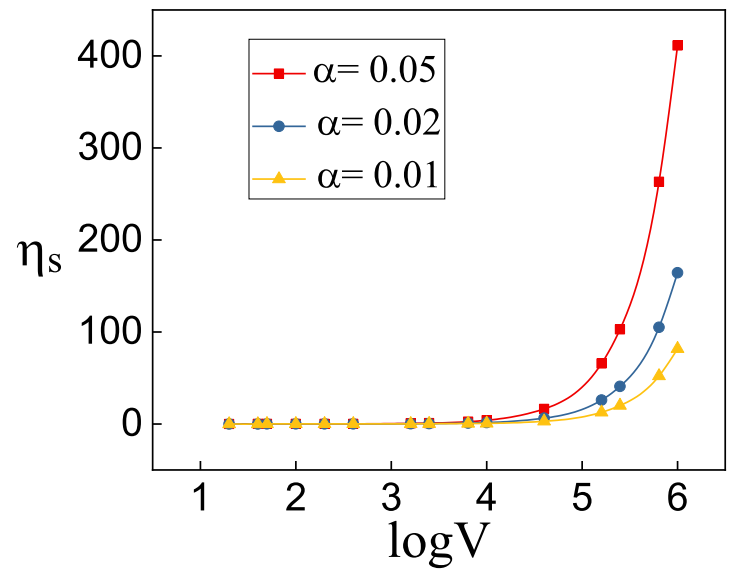

FIG. 7. Dynamic efficiency $\eta_{S}$ in normal oscillation regions for different system sizes $V$. In the normal oscillation region, the dynamic efficiency $\eta_{S}$ increases as the system size $V$ increases.

\section{Dynamics efficiency}

For $\alpha<0$ in the noise-induced oscillation region, based on Eqs. (C11) and (C12), one can calculate where the maximum value of $\eta_{S}$ is located when $D_{\theta} \simeq 6 \alpha$. Thus

$$
V_{\mathrm{opt}} \simeq-\frac{3 C_{r} \varepsilon^{2}}{4 \alpha^{2}}
$$

For $\alpha>0$ in the normal oscillation region, the plotted dynamic efficiency $\eta_{S}$ (Fig. 7) increases as the system size $V$ increases, which means that the optimal system size is absent for systems with sufficient energy supplies.

\section{APPENDIX D: DETAILS OF THE MODELS}

\section{Brusselator}

The Brusselator model is described in the main text. At the macroscopic limit, the deterministic reaction rate for species $X$ and $Y$ is given by

$$
\begin{gathered}
\frac{d x_{1}}{d t}=k_{1} A-k_{2} B x_{1}-k_{3} x_{1}+k_{4} x_{1}^{2} x_{2}, \\
\frac{d x_{2}}{d t}=k_{2} B x_{1}-k_{4} x_{1}^{2} x_{2},
\end{gathered}
$$

where $x_{1}$ and $x_{2}$ are the concentrations of $X_{1}$ and $X_{2}$, respectively. The rate constants are represented by $\boldsymbol{k}=$ $\left\{k_{1}, k_{2}, k_{3}, k_{4}\right\}$. Here, we set $k_{i}=1, \forall i$ without loss of generality. The unique steady state of the system is given by $\left(x_{1, s}, x_{2, s}\right)=(A, B / A)$, which loses stability when the control parameter $\boldsymbol{B}$ exceeds the Hopf bifurcation point $B_{c}=1+A^{2}$ where the limit cycle emerges. The behavior of the system near Hopf bifurcation can be described by the stochastic normal form equation on a two-dimensional center manifold based on the bifurcation theory. The parameters in stochastic normal form theory can be calculated by setting $A=1$, which reads $C_{r}=-3 / 8, C_{i}=-1 / 24, \varepsilon^{2} \simeq 4, \omega=1$, and $\alpha=\left(B-1-A^{2}\right) / 2$. 
TABLE I. Description of the circadian clock model.

\begin{tabular}{llll}
\hline \hline & Reaction & Transition rate & Biochemical function \\
\hline 1 & $\mathrm{G} \rightarrow \mathrm{R}+\mathrm{G}$ & $w_{1}=v_{s} k_{I}^{n}\left(k_{I}^{n}+x_{3}^{n}\right)^{-1}$ & transcription \\
2 & $\mathrm{R} \rightarrow$ & $w_{2}=v_{m} x_{z}^{n}\left(k_{m}+x_{1}\right)^{-1}$ & $\mathrm{R}$ degradation \\
3 & $\mathrm{R} \rightarrow \mathrm{R}+\mathrm{P}_{C}$ & $w_{3}=k_{s} x_{1}$ & translation \\
4 & $\mathrm{P}_{C} \rightarrow$ & $w_{4}=v_{d} x_{2}\left(k_{d}+x_{2}\right)^{-1}$ & degradation of $\mathrm{P}_{C}$ \\
5 & $\mathrm{P}_{C} \rightarrow \mathrm{P}_{R}$ & $w_{5}=k_{1} x_{2}$ & transport of $\mathrm{P}_{C}$ into the nucleus \\
6 & $\mathrm{P}_{N} \rightarrow \mathrm{P}_{C}$ & $w_{6}=k_{2} x_{3}$ & transport of $\mathrm{P}_{N}$ out of the nucleus
\end{tabular}

\section{Circadian clock}

The circadian clock model considered here (see Table I) incorporates the transcription of the gene $(\mathrm{G})$ involved in the biochemical clock and transport of the mRNA (R) into the cytosol, where it is translated into clock proteins $\left(\mathrm{P}_{C}\right)$ and degraded [47]. The protein can be degraded or transported into the nucleus $\left(\mathrm{P}_{N}\right)$, where it exerts a negative regulation on the expression of its gene. Here, we set $\boldsymbol{x}=\left(x_{1}, x_{2}, x_{3}\right)$ to stand for the concentrations of $\left(\mathrm{R}, \mathrm{P}_{C}, \mathrm{P}_{N}\right)$. The transcription rate of mRNA is chosen as the control parameter, represented by $v_{s}$. The parameters are $k_{I}=2.0 \mathrm{nM}$, Hill coefficient $n=4$, $v_{m}=0.3 \mathrm{nM} / \mathrm{h}, k_{m}=0.2 \mathrm{nM}, k_{s}=2.0 \mathrm{~h}^{-1}, v_{d}=1.5 \mathrm{nM} / \mathrm{h}$, $k_{d}=0.1 \mathrm{nM}$, and $k_{1}=k_{2}=0.2 \mathrm{~h}^{-1}$. Under such conditions, the Hopf bifurcation point locates at $v_{s} \simeq 0.25725$. In addition, parameter values used in the stochastic normal form theory can be calculated as $C_{r} \simeq-0.3474, C_{i} \simeq 0.5722$, and $\varepsilon^{2} \simeq 0.3556$.
[1] J. E. Ferrell, Jr., T. Y.-C. Tsai, and Q. Yang, Modeling the cell cycle: Why do certain circuits oscillate? Cell 144, 874 (2011).

[2] M. Nakajima, K. Imai, H. Ito, T. Nishiwaki, Y. Murayama, H. Iwasaki, T. Oyama, and T. Kondo, Reconstitution of circadian oscillation of cyanobacterial KaiC phosphorylation in vitro, Science 308, 414 (2005).

[3] A. Goldbeter, Biochemical Oscillations and Cellular Rhythms: The Molecular Bases of Periodic and Chaotic Behaviour (Cambridge University Press, Cambridge, 1997).

[4] J.-L. Martiel and A. Goldbeter, A model based on receptor desensitization for cyclic AMP signaling in Dictyostelium cells, Biophys. J. 52, 807 (1987).

[5] G. Buzsáki and A. Draguhn, Neuronal oscillations in cortical networks, Science 304, 1926 (2004).

[6] G. Lan, P. Sartori, S. Neumann, V. Sourjik, and Y. Tu, The energy-speed-accuracy trade-off in sensory adaptation, Nat. Phys. 8, 422 (2012).

[7] R. G. Endres and N. S. Wingreen, Maximum Likelihood and the Single Receptor, Phys. Rev. Lett. 103, 158101 (2009).

[8] G. Lan and Y. Tu, The cost of sensitive response and accurate adaptation in networks with an incoherent type-1 feed-forward loop, J. R. Soc. Interface 10, 20130489 (2013).

[9] P. Sartori and Y. Tu, Free Energy Cost of Reducing Noise while Maintaining a High Sensitivity, Phys. Rev. Lett. 115, 118102 (2015).

[10] T. Mora and I. Nemenman, Physical Limit to Concentration Sensing in a Changing Environment, Phys. Rev. Lett. 123, 198101 (2019).

[11] Y. Cao, H. Wang, Q. Ouyang, and Y. Tu, The free-energy cost of accurate biochemical oscillations, Nat. Phys. 11, 772 (2015).

[12] A. C. Barato and U. Seifert, Cost and Precision of Brownian Clocks, Phys. Rev. X 6, 041053 (2016).

[13] C. Fei, Y. Cao, Q. Ouyang, and Y. Tu, Design principles for enhancing phase sensitivity and suppressing phase fluctuations simultaneously in biochemical oscillatory systems, Nat. Commun. 9, 1434 (2018).
[14] Y. Hasegawa and T. Van Vu, Uncertainty relations in stochastic processes: An information inequality approach, Phys. Rev. E 99, 062126 (2019).

[15] H. Qian, Phosphorylation energy hypothesis: Open chemical systems and their biological functions, Annu. Rev. Phys. Chem. 58, 113 (2007).

[16] S. Guan, L. Xu, Q. Zhang, and H. Shi, Trade-offs between effectiveness and cost in bifunctional enzyme circuit with concentration robustness, Phys. Rev. E 101, 012409 (2020).

[17] Z. Hou, T. Rao, and H. Xin, Effects of internal noise for rate oscillations during CO oxidation on platinum surfaces, J. Chem. Phys. 122, 134708 (2005).

[18] T. Xiao, J. Ma, Z. Hou, and H. Xin, Effects of internal noise in mesoscopic chemical systems near Hopf bifurcation, New J. Phys. 9, 403 (2007).

[19] C. W. Gardiner, Handbook of Stochastic Methods: For Physics, Chemistry and the Natural Sciences, Springer Series in Synergetcs Vol. 13 (Springer, Berlin, 1985).

[20] Z. Hou and H. Xin, Internal noise stochastic resonance in a circadian clock system, J. Chem. Phys. 119, 11508 (2003).

[21] P. Hänggi, Stochastic resonance in biology: How noise can enhance detection of weak signals and help improve biological information processing, ChemPhysChem 3, 285 (2002).

[22] Z. Hou, T. J. Xiao, and H. Xin, Internal noise coherent resonance for mesoscopic chemical oscillations: A fundamental study, ChemPhysChem 7, 1520 (2006).

[23] J. Ma, T. Xiao, Z. Hou, and H. Xin, Coherence resonance induced by colored noise near Hopf bifurcation, Chaos 18, 043116 (2008).

[24] C. H. Ko, Y. R. Yamada, D. K. Welsh, E. D. Buhr, A. C. Liu, E. E. Zhang, M. R. Ralph, S. A. Kay, D. B. Forger, and J. S. Takahashi, Emergence of noise-induced oscillations in the central circadian pacemaker, PLoS Biol. 8, e1000513 (2010).

[25] U. Seifert, Entropy Production along a Stochastic Trajectory and an Integral Fluctuation Theorem, Phys. Rev. Lett. 95, 040602 (2005). 
[26] A. Dechant and S.-i. Sasa, Current fluctuations and transport efficiency for general Langevin systems, J. Stat. Mech. (2018) 063209.

[27] W. Hwang and C. Hyeon, Energetic costs, precision, and transport efficiency of molecular motors, J. Phys. Chem. Lett. 9, 513 (2018).

[28] A. C. Barato and U. Seifert, Thermodynamic Uncertainty Relation for Biomolecular Processes, Phys. Rev. Lett. 114, 158101 (2015).

[29] P. Pietzonka, A. C. Barato, and U. Seifert, Universal bound on the efficiency of molecular motors, J. Stat. Mech. (2016) 124004.

[30] J. M. Horowitz and T. R. Gingrich, Thermodynamic uncertainty relations constrain non-equilibrium fluctuations, Nat. Phys. 16, 15 (2020).

[31] Y. Hasegawa and M. Arita, Optimal Implementations for Reliable Circadian Clocks, Phys. Rev. Lett. 113, 108101 (2014).

[32] Y. Hasegawa and M. Arita, Circadian clocks optimally adapt to sunlight for reliable synchronization, J. R. Soc. Interface 11, 20131018 (2014); J. R. Soc., Interface 12, 20150374(E) (2015).

[33] E. D. Buhr, S.-H. Yoo, and J. S. Takahashi, Temperature as a universal resetting cue for mammalian circadian oscillators, Science 330, 379 (2010).

[34] D. Saunders, S. Gillanders, and R. Lewis, Light-pulse phase response curves for the locomotor activity rhythm in Period mutants of Drosophila melanogaster, J. Insect Physiol. 40, 957 (1994).

[35] C. H. Johnson, Phase response curves: What can they tell us about circadian clocks? in Circadian Clocks from Cell to Human: Proceedings of the Fourth Sapporo Symposium on Biological Rhythm (Hokkaido University Press, Sapporo, 1992), pp. 209-249.

[36] Y. Kuramoto, Chemical Oscillations, Waves, and Turbulence (Dover, Mineola, 2003).
[37] D. S. Goldobin, J.-N. Teramae, H. Nakao, and G. B. Ermentrout, Dynamics of Limit-Cycle Oscillators Subject to General Noise, Phys. Rev. Lett. 105, 154101 (2010).

[38] J. W. Shuai and P. Jung, Optimal Intracellular Calcium Signaling, Phys. Rev. Lett. 88, 068102 (2002).

[39] J. W. Shuai and P. Jung, Optimal ion channel clustering for intracellular calcium signaling, Proc. Natl. Acad. Sci. USA 100, 506 (2003).

[40] G. Schmid, I. Goychuk, and P. Hänggi, Stochastic resonance as a collective property of ion channel assemblies, Europhys. Lett. 56, 22 (2001).

[41] J. Zhang, Z. Hou, and H. Xin, System-size biresonance for intracellular calcium signaling, ChemPhysChem 5, 1041 (2004).

[42] B. Nguyen and U. Seifert, Exponential volume dependence of entropy-current fluctuations at first-order phase transitions in chemical reaction networks, Phys. Rev. E 102, 022101 (2020).

[43] L. Arnold, N. Sri Namachchivaya, and K. R. Schenk-Hoppé, Toward an understanding of stochastic Hopf bifurcation: A case study, Int. J. Bifurcation Chaos Appl. Sci. Eng. 6, 1947 (1996).

[44] D. Schnoerr, G. Sanguinetti, and R. Grima, The complex chemical Langevin equation, J. Chem. Phys. 141, 024103 (2014).

[45] D. T. Gillespie, The chemical Langevin equation, J. Chem. Phys. 113, 297 (2000).

[46] B. D. Hassard,, N. D. Kazarinoff, and Y.-H. Wan, Theory and Applications of Hopf Bifurcation, London Mathematical Society Lecture Note Series Vol. 41 (Cambridge University Press, Cambridge, 1981).

[47] T. Xiao, Z. Hou, and H. Xin, Stochastic thermodynamics in mesoscopic chemical oscillation systems, J. Phys. Chem. B 113, 9316 (2009). 